\title{
Frequência de síndrome metabólica em estudantes de uma universidade pública
}

\section{brasileira}

\author{
Frequency of metabolic syndrome in students at a Brazilian public university \\ Frecuencia del síndrome metabólico en estudiantes de una universidad pública brasileña
}

Recebido: 24/08/2021 | Revisado: 04/09/2021 | Aceito: 10/09/2021 | Publicado: 12/09/2021

\author{
Juscelino de Souza Borges Neto \\ ORCID: https://orcid.org/0000-0002-4551-3278 \\ Universidade Federal de São João Del Rei, Brasil \\ E-mail: celinoborges1980@gmail.com \\ Juliana Mara Flores Bicalho \\ ORCID: https://orcid.org/0000-0003-1445-8234 \\ Universidade Federal de São João Del Rei, Brasil \\ E-mail: jmfbicalho@gmail.com \\ Hiure Gomes Ramos Meira \\ ORCID: https://orcid.org/0000-0001-8563-3170 \\ Universidade Federal de São João Del Rei, Brasil \\ E-mail: hiureg100@gmail.com \\ Mariana Sousa Vieira \\ ORCID: https://orcid.org/0000-0002-2764-0863 \\ Universidade Federal de Minas Gerais, Brasil \\ E-mail: vieira.msousa@gmail.com \\ Daniel Bonoto Gonçalves \\ ORCID: https://orcid.org/0000-0002-8178-1026 \\ Universidade Federal de São João Del Rei, Brasil \\ E-mail: bonoto@ufsj.edu.br \\ José Antônio da Silva \\ ORCID: https://orcid.org/0000-0001-9134-1211 \\ Universidade Federal de São João Del Rei, Brasil \\ E-mail: zecsilva@yahoo.com.br \\ Maria Emília Soares Martins do Santos \\ ORCID: https://orcid.org/0000-0001-8922-0867 \\ Universidade Federal do Triângulo Mineiro, Brasil \\ E-mail: mariaemilia.santos@uftm.edu.br \\ Paulo Afonso Granjeiro \\ ORCID: https://orcid.org/0000-0003-0322-0861 \\ Universidade Federal de São João Del Rei, Brasil \\ E-mail:pagranjeiro@gmail.com
}

\begin{abstract}
Resumo
Introdução: Síndrome Metabólica (SM) é caracterizada por distúrbios metabólicos que geram obesidade central, hipertensão arterial sistêmica, dislipidemia e intolerância à glicose. A prevalência da SM entre jovens adultos é considerada baixa, no entanto seus componentes individuais têm aumentado de forma preocupante. A taxa de morbimortalidade decorrentes de doenças crônicas entre jovens é preocupante no Brasil e o diagnóstico precoce é importante por se tratar de uma síndrome silenciosa. Objetivo: Determinar a prevalência de SM em universitários de uma Instituição de Ensino Pública (IES). Métodos: estudo descritivo, amostra composta por 123 universitários de ambos os gêneros, com idade entre 18 e 22 anos. Foi utilizado questionário para informações socioeconômicas e sobre estilo de vida. A coleta de sangue e de dados antropométricos foi realizada na própria IES. Resultados: $69,1 \%$ eram do gênero feminino; $12,2 \%$ já tiveram contato com o fumo de forma ativa; $56,1 \%$ consumiram bebidas alcóolicas pelo menos uma vez nos últimos 30 dias; 67,47\% eram sedentários. 54,28\% estão acima do peso; 15,8\% dos homens encontram-se com relação cintura-quadril moderadamente alta; entre as mulheres 44,7\% estão com risco moderado; $10,6 \%$ de alto risco e $5,9 \%$ relação de muito alto risco. O parâmetro individual mais alterado foi o HDLc 30,9\%, enquanto o menos alterado foi a glicemia com $0,81 \%$. Conclusão: apesar da baixa frequência de SM observada no estudo houve considerável frequência de pelo menos um parâmetro alterado, com destaque para o TAG e HDLc, o que se faz necessário o diagnóstico precoce e o monitoramento para prevenir o desenvolvimento crônico da SM.
\end{abstract}

Palavras-chave: Síndrome metabólica; Estudantes; Universidade. 


\begin{abstract}
Introduction: Metabolic Syndrome (MS) is characterized by metabolic disorders that generate central obesity, systemic arterial hypertension, dyslipidemia and glucose intolerance. The prevalence of MS among young adults is considered low, however its individual components have been worryingly increasing. The morbidity and mortality rate resulting from chronic diseases among young people is worrying in Brazil and early diagnosis is important because it is a silent syndrome. Objective: To determine the prevalence of MS in university students of a Public Education Institution (IES). Methods: descriptive study, sample composed of 123 university students of both genders, aged between 18 and 22 years. A questionnaire was used for socioeconomic and lifestyle information. Blood collection and anthropometric data were performed at the IES itself. Results: $69.1 \%$ were female; $12.2 \%$ have had active contact with tobacco; $56.1 \%$ consumed alcoholic beverages at least once in the last 30 days; $67.47 \%$ were sedentary. $54.28 \%$ are overweight; $15.8 \%$ of men have a moderately high waist-hip ratio; among women, $44.7 \%$ are at moderate risk; $10.6 \%$ high risk and $5.9 \%$ very high risk ratio. The most altered individual parameter was HDLc 30.9\%, while the least altered was blood glucose with $0.81 \%$. Conclusion: despite the low frequency of MS observed in the study, there was a considerable frequency of at least one altered parameter, especially TAG and HDLc, which requires early diagnosis and monitoring to prevent the chronic development of MS.
\end{abstract}

Keywords: Metabolic syndrome; Students; University.

\title{
Resumen
}

Introducción: El Síndrome Metabólico (SM) se caracteriza por alteraciones metabólicas que generan obesidad central, hipertensión arterial sistémica, dislipidemia e intolerancia a la glucosa. La prevalencia de la EM entre los adultos jóvenes se considera baja, sin embargo, sus componentes individuales han aumentado de manera preocupante. La tasa de morbilidad y mortalidad por enfermedades crónicas entre los jóvenes es preocupante en Brasil y el diagnóstico precoz es importante porque se trata de un síndrome silencioso. Objetivo: Determinar la prevalencia de EM en estudiantes universitarios de una Institución de Educación Pública (IES). Métodos: estudio descriptivo, muestra compuesta por 123 estudiantes universitarios de ambos sexos, con edades comprendidas entre 18 y 22 años. Se utilizó un cuestionario para obtener información socioeconómica y de estilo de vida. La recogida de sangre y los datos antropométricos se realizaron en el propio IES. Resultados: el 69,1\% eran mujeres; El 12,2\% ha tenido contacto activo con el tabaco; 56,1\% consumió bebidas alcohólicas al menos una vez en los últimos 30 días; El 67,47\% eran sedentarios. El 54,28\% tiene sobrepeso; El $15,8 \%$ de los hombres tiene una relación cintura-cadera moderadamente alta; entre las mujeres, el $44,7 \%$ tiene un riesgo moderado; $10,6 \%$ de alto riesgo y 5,9\% de riesgo muy alto. El parámetro individual más alterado fue el cHDL $30,9 \%$, mientras que el menos alterado fue la glucemia con 0,81\%. Conclusión: a pesar de la baja frecuencia de EM observada en el estudio, hubo una frecuencia considerable de al menos un parámetro alterado, especialmente TAG y HDLc, que requiere un diagnóstico y seguimiento precoces para prevenir el desarrollo crónico de EM.

Palabras clave: El síndrome metabólico; Estudiantes; Universidad.

\section{Introdução}

A Síndrome Metabólica (SM) é caracterizada por distúrbios metabólicos individuais que geram obesidade central, hipertensão arterial sistêmica (HAS), dislipidemia e intolerância à glicose (Marco et al., 2012). A obesidade está substancialmente ligada à SM que por consequência aumenta a mortalidade total por doenças cardiovasculares (Aboufotouth et al., 2012). As doenças crônicas não transmissíveis e seus fatores de risco, incluindo a HAS e obesidade, são a principal causa de morbidade, mortalidade e incapacidade no mundo, e constituem um desafio complexo em saúde pública, além de grave ameaça ao desenvolvimento econômico e social (Gesteiro, 2021; Bicalho et al., 2021a). Os números de morbimortalidade e custos socioeconômicos aos serviços públicos de saúde associados à SM são alarmantes e sua prevalência varia conforme critério de diagnóstico (Borges Neto et al., 2021).

Os componentes da Síndrome Metabólica são: hipertensão arterial sistêmica (HAS), obesidade central, juntamente com os elementos sanguíneos glicose, triglicérides (TAG) e colesterol de alta densidade (HDLc; Eckel e Zimmet., 2006). Estes componentes interferem diretamente no surgimento de complicações cardiovasculares (Pereira, 2012). A etiologia da SM não é clara por ter origens multivariadas. Dentre as possíveis causas do surgimento dos seus componentes estão os hábitos alimentares inadequados (Oliveira et al., 2009), maior probabilidade de desenvolver SM pessoas que sofrem estresse no trabalho, os obesos, sedentários com padrões dietéticos hipercalóricos e fatores genético desfavoráveis (Freitas et al., 2012), assim como pessoas que possuem resistência à insulina e intolerância à glicose (Morrell et al., 2012), assim como em pacientes esquizofrênico com 
influência de polimorfismo nos genes ANKK1, DRD2 e DRD3 (Pinto et al., 2018). Esses hábitos de vida vêm contribuindo com a prevalência de SM desde a infância (Granjeiro et al., 2016; Gonçalves et al., 2021).

Com o avanço da ciência e tecnologia em prol da saúde, do conforto e da comodidade, tivemos um aumento da expectativa de vida, porém os níveis de atividades físicas realizadas quando efetuando as tarefas do cotidiano diminuíram, e com isso, as manifestações dos componentes da SM têm aumentado, tornando-se uma preocupação para a saúde pública por gerar altos índices de morbimortalidade (Siqueira et al., 2006). Além do mais, mudanças dos hábitos alimentares têm tornado as pessoas cada vez mais obesas (Lopes, 2007). O comportamento alimentar está relacionado tanto com aspectos técnicos e objetivos como o alimento, o momento e o lugar que comemos, mas também com aspectos socioculturais e psicológicos (Bicalho et al., 2021b).

Somam-se a isto que, no ambiente competitivo atual, estudantes universitários enfrentam pressão relacionada aos estudos, provas, colegas, professores e familiares e muitas vezes experimentam altas quantidades de estresse que exerce efeito negativo no desempenho acadêmico, na saúde emocional e do corpo. Isso tem demonstrado associação com o surgimento de patologias entre os estudantes universitários, especialmente da área da saúde, aumentando o risco para doenças crônicas não transmissíveis (Huang et al., 2007; Oviedo et al., 2008; Smith \& Essop. 2009; Jesus et al., 2020). Almejando um bom desempenho acadêmico, boas relações sociais e culturais, os universitários muitas vezes trocam as refeições completas por lanches práticos, com alto valor calórico e baixa relação nutricional (Santos et al., 2014; Evaristo et al., 2021). A elevada ingestão de arroz, batata chips e panquecas estão associadas à presença da SM (DiBello et al., 2009), assim como arroz, feijão, gordura, sobremesas lácteas, bebidas e doces açucarados estão relacionados também à SM e a baixa concentração de HDLc sanguíneo (Noel et al., 2009).

A prevalência da SM entre jovens adultos é considerada baixa, no entanto a de seus componentes individuais têm aumentado de forma preocupante. A taxa de morbimortalidade decorrentes de doenças crônicas nos extratos etários mais jovens é preocupante no Brasil e também em outros países (Barbalho et al., 2009). O diagnóstico precoce é importante por se tratar de uma síndrome silenciosa. Portanto, objetivou-se nesse estudo avaliar a frequência dos componentes da SM em estudantes de uma Universidade Pública de Divinópolis/MG, utilizando como critério de diagnóstico o preconizado pela NCEP/ATP/III.

\section{Métodos}

Trata-se de um estudo descritivo transversal (Pereira, 2011), composto por 123 estudantes universitários com idades entre 18 e 22 anos, de uma Instituição de Ensino Superior Pública (IES) da cidade de Divinópolis - MG, amostra calculada com uso de fórmula para população finita.

Os critérios de inclusão foram: estar matriculado na IES, estar na faixa etária entre 18 e 22 anos, aceitar participar do estudo e de todas suas etapas (entrevista e coleta de sangue) e assinar o termo de consentimento livre esclarecido.

A coleta ocorreu em dois momentos, sendo realizada de março a novembro de 2011. No primeiro momento foi aplicado um questionário para angariar informações socioeconômicas e sobre o estilo de vida (tabagismo, etilismo e prática de atividade física), além da coleta de sangue para determinação dos componentes sanguíneos da SM: glicose, TAG e HDLc. No segundo momento foram coletados os dados antropométricos, pressão arterial (PA), circunferência da cintura (CC), circunferência do quadril (CQ), estatura (m), índice de massa corporal (IMC) e relação cintura quadril (WHR/RCQ) conforme metodologia realizada por Teixeira et al. (2014).

Para os resultados quanto ao IMC foi avaliada o peso corporal $(\mathrm{Kg})$ e a estatura $(\mathrm{m})$ e em seguida calculado pela fórmula $\mathrm{Kg} / \mathrm{m}^{2}$. Foram classificados com peso normal IMC < 25, sobrepeso IMC entre 25 e 29.99, obesidade I IMC entre 30 e 34.99 , obesidade II IMC > 35. Para a RCQ/WHR, foi aferida com fita métrica em cm, entre a parte mais inferior do rebordo costal e a 
crista ilíaca anterossuperior, a menor circunferência da cintura, e o quadril $(\mathrm{cm})$ a maior circunferência entre a crista ilíaca e o trocânter maior. A WHR se dá pelo resultado da divisão entre a circunferência da cintura/ pela circunferência do quadril. Os valores de referência quanto ao risco baixo, moderado, alto e muito alto para homens são: < 0.83 ; entre 0.83 e $0.88 ; 0.89$ e 0.94 e >0.94 respectivamente, já para as mulheres considerando os mesmos riscos os valores são < 0.71 ; entre 0.71 e 0.77 ; 0.78 e 0.82 e >0.82 respectivamente (Associação Brasileira para o Estudo da Obesidade e da Síndrome Metabólica [ABESO], 2009).

O National Cholesterol Education Program's Adult Treatment Panel III (NCEP-ATP III) foi utilizado como critério de diagnóstico para os componentes da SM. Portanto, utilizando como valores de corte para $\mathrm{CC}$ de $>88 \mathrm{~cm}$ e $>102 \mathrm{~cm}$ para mulheres e homens, respectivamente; PA de $\geq 130 / 80$ ou diagnóstico de hipertensão; TG de $\geq 150 \mathrm{mg} / \mathrm{dL} ; \mathrm{HDLc}$ de $\leq 40 \mathrm{mg} / \mathrm{dL}$ para homens $\mathrm{e} \leq 50$ para mulheres e glicose de $\geq 100 \mathrm{mg} / \mathrm{dL}$ ou diagnóstico de diabetes (Expert Panel on Detection, Evaluation, and Treatment of High Blood Cholesterol in Adults, 2001). Para os exames laboratoriais sugeriu-se 12 horas de jejum, as coletas foram executadas na própria universidade.

Em relação ao estilo de vida, considerando um mês anterior ao preenchimento do questionário, foram classificados como fumantes os que fumavam todos os dias um cigarro no mínimo e fumantes ocasionais (fumam, mas não fuma todos os dia); foram considerado etilistas os que bebiam no mínimo três vezes por semana. Foram considerados sedentários os indivíduos que praticavam atividades físicas menos de três vezes por semana, trinta minutos por sessão (Ministério da Saúde [MS], 2010).

O programa Statistical Package for the Social Sciences IBM (SPSS), versão 20.0 foi utilizado para calcular as médias, qui-quadrado exato de Fisher, assim como as proporções e teste $t$ de Student. Foram consideradas significativas e inferenciais somente aquelas análises estatísticas as quais o valor de $\mathrm{p}<0,05$.

O projeto foi aprovado pelo Comitê de Ética em Pesquisa (CEP) da Universidade Federal de São João Del-Rei, Campus Centro Oeste, de acordo com CAAE nº 19436213.6.0000.5545.

\section{Resultados}

Participaram do estudo 123 estudantes universitários com idade entre 18 e 22 anos. 30,9\% eram do gênero masculino e $69,1 \%$ feminino; $53,66 \%$ brancos e $46,43 \%$ não brancos.

No Quadro 1 estão apresentados os dados antropométricos e também os relativos ao estilo de vida dos estudantes universitários de Divinópolis - MG. A maioria dos homens eram fisicamente ativos (60,5\%), enquanto entre as mulheres $80 \%$ foram consideradas sedentárias. Com relação ao fumo, 96,5\% e 97,3\% das mulheres e homens, respectivamente, não fumavam. Quanto ao etilismo, 92,2\% dos homens e 96,5\% mulheres não beberam mais de duas vezes por semana nos últimos trinta dias. Só houve diferença estatisticamente significativa entre homens e mulheres quanto a prática de atividade física.

Pode-se observar que apesar da maioria $(44,71 \%)$ estar com IMC normal, uma considerável parcela estava acima do peso $(40,65 \%)$. Nenhuma mulher foi observada com obesidade II, ao passo que para os homens essa prevalência foi de 2,63\%. A RCQ entre as mulheres foi encontrada o risco baixo em 35,3\%, enquanto que o risco moderado acometeu 45,90\%. Para o risco alto e muito alto, a prevalência foi de $11,76 \%$ e $7,06 \%$, respectivamente. O risco baixo e moderado da RCQ, para os homens, foi de $84,21 \%$ e $15,80 \%$, respectivamente, e não houve prevalência de risco alto nem de risco muito alto. Os valores de IMC entre

homens e mulheres não apresentaram diferença estatisticamente significativa, no entanto, a proporção de mulheres com RCQ com risco baixo e moderado foi estatisticamente significativa quando comparadas aos homens. 
Research, Society and Development, v. 10, n. 12, e37101219802, 2021 (CC BY 4.0) | ISSN 2525-3409 | DOI: http://dx.doi.org/10.33448/rsd-v10i12.19802

Quadro 1 - Distribuição do estilo de vida e dados antropométricos dos universitários, Divinópolis - MG, Brasil.

\begin{tabular}{|c|c|c|c|c|}
\hline Variáveis & $\begin{array}{c}\mathrm{N}=123 \% \\
(\mathrm{IC})\end{array}$ & $\begin{array}{c}\text { Masculino }=38 \% \\
\text { (IC) }\end{array}$ & $\begin{array}{c}\text { Feminino }=85 \% \\
\text { (IC) }\end{array}$ & $* P$-valor \\
\hline Atividade Física & & & & $<0.001$ \\
\hline Sedentário & $67.8(58.4-75.6)$ & $39.5(24-56.6)$ & $80(69.9-87.9)$ & \\
\hline \multicolumn{5}{|l|}{ Tabagismo } \\
\hline Sim & $3.2(0.9-8.1)$ & $2.6(0.06-13.8)$ & $3.6(0.7-9.9)$ & \\
\hline \multicolumn{5}{|l|}{ Etilismo } \\
\hline Não & $95.1(89.7-98.2)$ & $92.1(78.6-98.3)$ & $96.4(90-99.2)$ & 0.5 \\
\hline Sim & $4.9(1.8-10.3)$ & $7.9(1.65-21.39)$ & $3.6(0.7-9.9)$ & \\
\hline Obesidade I $(30-34,99)$ & $13.8(8.26-21.2)$ & $15.3(8.4-24.73)$ & $10.52(2.94-25.81)$ & 0.6 \\
\hline Obesidade II $(>35,00)$ & $0.82(0.2-4.44)$ & $0.0(0.0-4.24)$ & $2.63(0.06-13.81)$ & 0.6 \\
\hline \multicolumn{5}{|l|}{ Risco $\mathbf{R C Q}^{\mathrm{b}}$} \\
\hline Baixo & 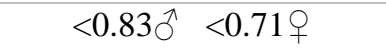 & $35.3(25.2-46.4)$ & $84.21(68.75-94)$ & $<0.001$ \\
\hline Moderado & $0.83-0.88$ ๙ $0.71-0.77 q$ & $45.9(35-57.04)$ & $15.8(6.02-31.25)$ & $<0.001$ \\
\hline Alto & $0.89-0.94 \overbrace{}^{\wedge} \quad 0.78-0.82$ q & $11.76(5.8-20.6)$ & $0.0(0.0-9.25)$ & 0.06 \\
\hline Muito alto & $>0.94 \widehat{\sigma}>0.82 q$ & $7.06(2.6-14.73)$ & $0.0(0.0-9.25)$ & 0.2 \\
\hline
\end{tabular}

*qui-square, a IMC Índice de Massa Corporal, b Relação Cintura Quadril. Fonte: Autores (2021). 
No Quadro 2 estão demonstrados os dados dos componentes da SM e o peso corporal relativos aos gêneros da amostra. Com relação à massa corporal e os componentes da SM dos estudantes, os homens apresentaram maiores médias em relação às mulheres quanto ao peso corporal, CC, PA e glicemia. Por outro lado, as mulheres apresentaram maiores médias para TAG. Quanto ao HDLc, as mulheres apresentaram melhores valores do que os homens. Quando comparadas as médias do peso e dos componentes da SM entre os gêneros, houve diferença estatisticamente significativa para glicemia $(p<0.05)$, para a massa corporal, CC, PAS, PAD e HDLc $(<0.001)$.

Quadro 2 - Dados antropométricos e componentes da Síndrome Metabólica (NCEP/ATP/III, 2001) entre estudantes de uma universidade pública. Divinópolis - MG, Brasil.

\begin{tabular}{|c|c|c|c|c|}
\hline Variáveis & $\begin{array}{c}\text { Total } \\
(\mathbf{N}=123) \\
\text { Mean } \pm \text { SD }^{\mathbf{a}}\end{array}$ & $\begin{array}{c}\text { Feminino } \\
(\mathbf{N}=\mathbf{8 5}) \\
\text { Mean } \pm \mathrm{SD}^{\mathrm{a}}\end{array}$ & $\begin{array}{c}\text { Masculino } \\
(\mathbf{N}=38) \\
\text { Mean } \pm \text { SD }^{\mathbf{a}}\end{array}$ & $p$-valor* \\
\hline Peso Corporal & $61.49 \pm 10.83$ & $58.21 \pm 9.16$ & $68.85 \pm 10.76$ & $<0.001$ \\
\hline Circunferência da Cintura (CC) & $72.40 \pm 7.38$ & $70.69 \pm 6.58$ & $76.24 \pm 7.71$ & $<0.001$ \\
\hline Pressão Arterial Sistólica (PAS) & $105.75 \pm 9.8$ & $103.57 \pm 9.46$ & $110.61 \pm 8.84$ & $<0.001$ \\
\hline Pressão Arterial Diastólica (PAD) & $66.12 \pm 11.25$ & $64.85 \pm 10.3$ & $68.96 \pm 12.84$ & 0.063 \\
\hline Glicemia & $76.56 \pm 14.06$ & $77.43 \pm 14.06$ & $81.41 \pm 11.21$ & $<0.05$ \\
\hline Triglicérides (TAG) & $87.13 \pm 39.89$ & $89.81 \pm 40.58$ & $81.13 \pm 38.16$ & 0.267 \\
\hline HDLc & $50.63 \pm 9.97$ & $53.85 \pm 9.08$ & $43.42 \pm 11.38$ & $<0.001$ \\
\hline
\end{tabular}

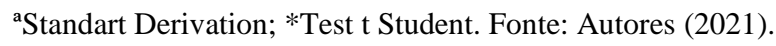

No Quadro 3 está apresentada a prevalência de alteração dos componentes individuais da SM de acordo com o critério da NCEP/ATP/III. O parâmetro individual mais alterado foi para HDLc, em ambos os gêneros, sendo superior para os homens. O segundo mais alterado foi TAG, seguido da PA e a CC. O componente menos alterado foi para glicemia. No entanto, quando comparamos os gêneros, os homens apresentaram maior prevalência do que as mulheres quanto à PA e à glicemia, e as mulheres obtiveram valores mais alterados do que os homens quanto à $\mathrm{CC}$ e ao TAG. Somente PAS e PAD obtiveram diferença estatisticamente significativa entre os gêneros. A prevalência de SM foi de $0.81 \%$, sendo de $2.63 \%$ para os homens e zero para as mulheres, não havendo diferença estatisticamente significativa entre eles. $61.8 \%$ não possui nenhum dos parâmetros da SM alterado, ao passo que $31.7 \%$ apresentaram um componente alterado, 5.7\% tiveram dois componentes alterados e com três parâmetros alterados foram encontrado $0.81 \%$ da amostra estudada. Não houve participante do estudo que apresentou quatro ou cinco parâmetros da SM alterados. Também não foi encontrada significância estatística quanto ao número de componentes da SM entre os gêneros. 
Quadro 3 - Prevalência de componentes alterados da Síndrome Metabólica e número de alterações da Síndrome Metabólica entre estudantes de uma universidade pública. Divinópolis - MG, Brasil.

\begin{tabular}{|c|c|c|c|c|}
\hline & $\begin{array}{c}\text { Total } \\
(\mathrm{N}=123) \\
\%(95 \% \mathrm{CI})\end{array}$ & $\begin{array}{c}\text { Feminino }(\mathbf{N}=\mathbf{8 5}) \\
\%(95 \% \mathrm{CI})\end{array}$ & $\begin{array}{c}\text { Masculino }(\mathbf{N}=38) \\
\%(95 \% \mathrm{CI})\end{array}$ & p-valor* \\
\hline \multicolumn{5}{|l|}{$\begin{array}{l}\text { Componentes } \\
\text { alterados }\end{array}$} \\
\hline $\begin{array}{c}\mathrm{CC} \\
\left(88 \mathrm{~cm}+102 \mathrm{~cm} \delta^{\Uparrow}\right)\end{array}$ & $1.62(0.2-5.75)$ & $2.35(0.29-8.24)$ & $0.0(0.0-9.25)$ & 0.4 \\
\hline $\begin{array}{l}\text { Hipertensão Arterial } \\
\qquad(\geq 130 / 80)\end{array}$ & $4(1.33-9.23)$ & $1.17(0.03-6.38)$ & $10.52(2.94-24.81)$ & $<0.05$ \\
\hline $\begin{array}{c}\text { Glicemia } \\
(\geq 100 \mathrm{mg} / \mathrm{dL})\end{array}$ & $0.81(0.02-4.44)$ & $0.0(0.0-4.24)$ & $2.63(0.06-13.81)$ & 0.3 \\
\hline $\begin{array}{c}\text { TAG } \\
(\geq 150 \mathrm{mg} / \mathrm{dL})\end{array}$ & $8.13(3.96-14.4)$ & $8.23(3.4-16.23)$ & $7.9(1.66-21.38)$ & 0.6 \\
\hline $\begin{array}{c}\text { HDLc } \\
(\leq 50 \stackrel{\circ}{+}, \leq 40 \text { ○) }\end{array}$ & $30.9(22.9-39.9)$ & $25.9(17-36.52)$ & $42.1(26.31-59.18)$ & 0.05 \\
\hline \multicolumn{5}{|l|}{ Número de alterações } \\
\hline 0 & $61.8(52.6-70.4)$ & $52.6(35.8-69)$ & $65.9(54.8-75.8)$ & 0.1 \\
\hline 1 & $31.7(23.6-40.7)$ & $34.2(19.6-31.3)$ & $30.6(21-41.5)$ & 0.6 \\
\hline 2 & $5.7(2.3-11.4)$ & $10.5(2.9-24.8)$ & $3.5(0.7-10)$ & 0.2 \\
\hline 3 & $0.81(0.2-4.4)$ & $2.6(0 ; 06-13.8)$ & $0.0(0.0-4.2)$ & 0.6 \\
\hline
\end{tabular}

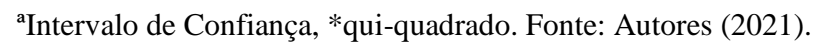

\section{Discussão}

Nessa amostra representativa de 123 estudantes universitários com idade de 18 a 22 anos, de Divinópolis - MG, onde $61,9 \%$ eram do gênero feminino, a prevalência de SM encontrada foi considerada baixa, perfazendo $0,81 \%$ dos participantes, em conformidade com o critério de diagnóstico NCEP ATP III. Na literatura, encontramos prevalência de SM variando entre 0,70\% (Huang et al., 2007), de acordo com o critério IDF (International Diabetes Federation) e 12,9\% (Topè \& Rogers, 2013), de acordo com NCEP ATP III, provavelmente devido ao perfil da população estudada e o critério de diagnóstico adotado.

Na cidade de Picos no estado do Piauí - Brasil, foram avaliados 550 estudantes universitários com idade média de 22,6 anos, e a prevalência de SM encontrada foi de 3,5\%, de acordo com o critério de diagnóstico NCEP ATP III (Silva et al., 2014). Em outro estudo realizado nos EUA (Fernandes \& Lofgren, 2011) com 189 graduandos com idade entre 18 e 24 anos, revelouse a prevalência de SM de 3,7\%, NCEP ATP III. No Irã (Rashid et al., 2012), também em conformidade com o NCEP ATP III, ao avaliar 221 universitários com idade entre 19 e 27 anos encontrou-se a prevalência de SM de 3,2\%. Duas pesquisas que obtiveram os resultados mais divergentes, ambas norte-americanas, a primeira desenvolvido em 2013 com 357 universitários, com idade entre 18 e 24 anos demonstrou prevalência de SM de 12,9\% (NCEP ATP III) (Topè \& Rogers, 2013), e a segunda realizada em 2007, utilizando o critério de diagnóstico da IDF, a amostra era composta por 300 estudantes universitários, com idade entre 18 e 24 anos demonstrou prevalência de SM de 0,7\% (Huang et al., 2007), ou seja, de acordo com o perfil da amostra, com região do país e do critério de diagnostico adotado, a prevalência de SM pode variar significativamente, no entanto, apenas o último estudo apresentou prevalência menor que este.

Com relação à composição corporal, dentre os universitários de Divinópolis - MG, 55,28\% estavam acima do peso com IMC entre 25,00 e 29,99 kg/m². Esse índice foi superior ao estudo desenvolvido com 501 estudantes universitários na Arábia 
Saudita, que, com IMC entre os mesmos valores, a prevalência foi de 21,9\% (Aboufotouth el al., 2012). No trabalho de Santos et al (2019) com 47 universitários do curso de Bacharel em Educação Física, em que a relação do peso corporal foi autorrelatada, 41,7\% afirmaram estar acima do peso, 37,5\% alegaram estar tentando perder peso, 35,4\% afirmaram estar fazendo dieta para redução de peso. Com o IMC entre 30,00 e $34,99 \mathrm{~kg} / \mathrm{m}^{2}$, denominado obesidade I, o presente estudo registrou 40,65\% dos participantes, e por último, 0,80\% estava com obesidade II (IMC > 35,00 kg/m²). No estudo saudita, com o IMC > 30,00 kg/m² foram encontrados 20,6\% da amostra, resultado inferior ao encontrado nesse estudo (Aboufotouth et al., 2012).

As mulheres do presente estudo apresentaram IMC de 26,1 $\pm 3,6$ (M \pm DP), 60\% delas estavam com sobrepeso, enquanto

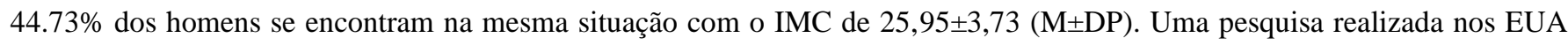
com 2103 universitários demonstrou resultado muito inferior ao aqui encontrando, pois 34,9\% e 21,1\% dos homens e mulheres respectivamente, estavam classificados como sobrepeso e 12,0\% dos homens e 6,1\% das mulheres estavam obesos (Morrell et al., 2012). No Irã (Rashid et al., 2012), os valores de IMC para as mulheres e os homens foram inferiores aos aqui verificados, $21,6 \pm 2,9(\mathrm{M} \pm \mathrm{DP})$ e $22,7 \pm 3,7(\mathrm{M} \pm \mathrm{DP})$ respectivamente.

Tratando da relação cintura quadril (RCQ), as mulheres apresentaram o índice de 0,72 $\pm 0,05$ (M $\pm \mathrm{DP}$ ) enquanto nos homens a RCQ foi 0,78 $\pm 0,04(\mathrm{M} \pm \mathrm{DP})$. Dois estudos apresentaram valores superiores aos aqui encontrados, quanto à WHR. Na África do Sul, um estudo conduzido com 266 estudantes em idade média de 21,5 anos, verificou-se que a RCQ feminina estava

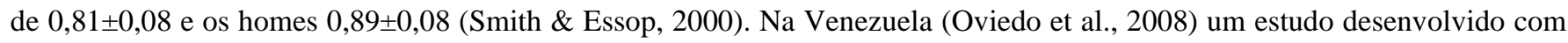
120 universitários com idade entre 18 e 26 anos, demonstrou que a RCQ das mulheres foi de 0,76 7,546 e entre os homens de $0,83 \pm 6,069$. Ambos os estudos apresentaram valores superiores aos encontrados nesse.

$\mathrm{Na}$ literatura encontramos valores muito diferentes para o mesmo componente da SM. O presente estudo demonstrou que a circunferência da cintura esteve prevalente em 1,62\% da amostra total. Resultado inferior aos obtidos em dois estudos conduzidos no nordeste brasileiro, o primeiro em Fortaleza - CE (Freitas et al., 2012), em que participaram 702 universitários com idade média de 21,5 anos, e o segundo desenvolvido em Picos- PI (Silva et al., 2014) com 550 graduandos com idade média de 22,6 anos. O estudo de Picos - PI apresentou 5,8\% enquanto o de Fortaleza - CE estava com 5,4\% da amostra com CC alterados. Ambos os estudos utilizaram o NCEP ATP III como critério de diagnóstico. Já nos EUA (Topè \& Rogers, 2013), também em conformidade com o NCEP ATP III, a prevalência de CC foi de 21,3\%. Quanto ao gênero, as mulheres do presente estudo estiveram prevalentes quanto à $\mathrm{CC}$ em 2,35\% enquanto nenhum dos homens apresentou a $\mathrm{CC}$ alterada. $\mathrm{O}$ estudo iraniano (Rashid et al., 2012) mostrou que ambos os homens e as mulheres estavam com a CC alterada em 1,8\%. Outro estudo demonstrou prevalência 5,2\% para homens e 4,2\% para mulheres (Morrell et al., 2012), ou seja, os valores encontrados são superiores aos deste estudo e variam de acordo com o perfil da amostra.

A hipertensão arterial sistêmica esteve presente em 4\% dos universitários desse estudo. Valor superior ao encontrado no estudo de Fortaleza (Freitas et al., 2012), que apresentou 3\% da amostra com PA alterado. No entanto, no estudo iraniano (Rashid et al., 2012), a PA esteve alterada em 16,7\% dos participantes, dentre eles 13,1\% do gênero feminino e 3,6\% masculino, o contrário do que ocorreu no presente estudo, pois as mulheres apresentaram menor prevalência $(1,17 \%)$ em relação aos homens $(10,52 \%)$.

A glicemia foi o parâmetro menos alterado entre os universitários de Divinópolis - MG, ocorrendo em 0,81\%, ou seja, um homem (2,63\%) apresentou o quadro de hiperglicemia, e todas as mulheres estavam com o índice glicêmico normal. Porém, na literatura, grande parte dos estudos mostra que a glicemia está alterada em valores consideráveis entre os jovens universitários. No estudo iraniano (Rashid et al., 2012) a prevalência de hiperglicemia foi de 9,0\% e no norte-americano (Topè \& Rogers, 2013), 22,1\%. Em Marília - SP (Barbalho et al., 2009), uma pesquisa desenvolvida com 67 universitários de 18 a 45 anos demonstrou que a hiperglicemia esteve prevalente em 8,2 e 16,7\% das mulheres e homens respectivamente. Outro estudo conduzido nos EUA (Morrell et al., 2012) mostrou que 13,7\% dos homens e 6,4\% das mulheres estiveram com a glicose alterada. 
Os dois componentes que estiveram mais alterados entre a amostra deste estudo foram os triglicérides com $8,13 \%$ de forma geral, entre as mulheres $8,23 \%$ e entre os homens 7,9\%, e o colesterol de alta densidade HDLc 30,9\% da amostra geral, 25,9 e $42,1 \%$ entre as mulheres e os homens respectivamente. Em concordância, a literatura correspondente mostra que os componentes da SM mais alterados entre os jovens universitários são os TAG e HDLc. No estudo desenvolvido em Fortaleza CE (Freitas et al., 2012), 23\% dos participantes estavam com os triglicérides alterados e 12\% com o HDLc. Em Picos - PI (Silva et al., 2014), 18,9 e 64,5\% da amostra estavam com TAG e HDLc alterados, respectivamente. Em Marília - SP (Barbalho et al., 2009), 33,3\% dos homens e 16,3\% das mulheres estavam com o TAG alterado, e considerando o valor de referência para HDLc $<50$ tanto para os homens quanto para as mulheres, 83,3\% dos homens e 49,0\% das mulheres estavam com HDLc alterado. Em outros países também é visível a maior prevalência desses componentes entre os universitários. Nos EUA (Topè \& Rogers, 2013), 11.2 e $37.3 \%$ estiveram com o TAG e HDLc alterados, nessa ordem. No Iran (Rashid et al., 2012), 14.1\% estavam com o TAG alterado, $10 \%$ das mulheres e $4.1 \%$ dos homens. Já com o HDLc alterado havia 26.2\% da amostra, $17.6 \%$ das mulheres e $8.6 \%$ dos homens. Portanto, esses resultados demonstram claramente que a dislipidemia está consideravelmente presente entre jovens universitários e provavelmente está vinculada ao estilo de vida dessas pessoas.

Relacionando o tabagismo, etilismo e atividade física, a presente pesquisa registrou que $96.7 \%$ dos universitários de Divinópolis - MG não faziam uso do tabaco, dentre eles 97.3 e $96.5 \%$ dos homens e mulheres respectivamente. Não foi encontrado nenhum resultado melhor que o do presente estudo. Dados encontrados na Venezuela (Oviedo et al., 2008) mostram que $65.83 \%$ da amostra nunca fumaram, e dentre eles $57.7 \%$ eram homens e $72.1 \%$ mulheres. Na cidade de Picos - PI (Silva et al., 2014), a amostra de $91.8 \%$ nunca fumou e também uma pesquisa conduzida nos EUA (Morrell et al., 2012) demonstrou que 93.1\% dos homens e 95,1\% das mulheres universitárias nunca fumaram. Quanto ao etilismo, 95.1\% da amostra não ingeriram bebida alcóolica 3 ou mais vezes por semana nos últimos 30 dias, dentre eles $92.1 \%$ dos homens e $96.5 \%$ das mulheres e $0.8 \%$ não respondeu sobre este assunto. Índices inferiores foram encontrados no estudo venezuelano (Oviedo et al., 2008), onde $68.33 \%$ da amostra bebeu pelo menos um drinque por semana no último mês, dentre eles $88.4 \%$ eram homens e $52.9 \%$ mulheres. Nos EUA (Morrell et al., 2012) também foram encontrados resultados mais baixos quanto ao etilismo em relação ao presente estudo. Entre os homens, $74.7 \%$ beberam pelo menos um drinque por semana no último mês e $60.8 \%$ das mulheres também beberam. A atividade física foi o item mais preocupante com relação ao estilo de vida. Somente $32.5 \%$ da amostra praticavam atividade física com intensão 3 ou mais vezes, durante 30 minutos, por semana nos últimos 90 dias. Os homens eram estatisticamente em maior número que as mulheres quanto à prática de atividade física, correspondendo $60.5 \%$ e as mulheres eram $20 \%$ da amostra. Já o sedentarismo foi prevalente em $67.5 \%$ da amostra, dentre eles 39.5\% dos homens e $80 \%$ das mulheres. Com relação ao estilo de vida, somente as proporções relacionadas à prática de atividade física, quando comparados os homens e mulheres houve significância estatística. Assim como no presente estudo, a literatura correspondente confirma os altos índices de sedentarismo entre os estudantes universitários, e ainda quanto ao gênero, às mulheres, na maioria das vezes, compondo a parte maior quanto ao sedentarismo. No estudo de Picos - PI (Silva et al., 2014) foi encontrada a prevalência de sedentarismo no valor de $71.7 \%$ da amostra, outro estudo desenvolvido em Fortaleza - CE (Pereira, 2012) com 702 estudantes universitários com idade entre 16 e 58 anos mostrou que $70.5 \%$ da amostra total estavam sedentárias e, compondo esse grupo estavam $59 \%$ dos homens e $77.4 \%$ das mulheres. Num estudo realizado na Venezuela (Oviedo et al., 2008) foi confirmado que $72.5 \%$ da amostra total éramos sedentárias e, dentre eles 73.1 e $72.1 \%$ dos homens e mulheres respectivamente. Todos estes estudos demonstraram resultados mais altos em relação à presente pesquisa, e esses valores são preocupantes e alarmantes, pois é sabido que o exercício físico realizado com intensão tem ação benéfica sobre todos os componentes da SM.

Quanto ao número de componentes da SM alterados, conforme o critério de diagnóstico do NCEP/ATP/III, a amostra que apresentou 1 componente alterado foi de 31.7\% e com 2 componentes alterados havia 5.7\%. Quanto ao gênero, os homens foram superiores às mulheres, sendo que eles apresentaram 34.2 e $10.5 \%$ para 1 e 2 componentes da SM alterados enquanto as 
mulheres apresentaram 30.6 e 3.5\% respectivamente. Utilizando o mesmo critério de diagnóstico, o estudo de Picos - PI (Silva et al., 2014) apresentou prevalência de um componente de $64.4 \%$ e com dois componentes alterados foram encontrados $11.6 \%$ da amostra, valores muito superiores aos do presente estudo. Outros dois estudos apresentaram prevalência de um componente semelhante ao presente estudo, 31.4\% (Topè \& Rogers, 2013) e 30.4\% (Freitas et al., 2012), no entanto, quanto a dois componentes da SM alterados o primeiro resultou em $20.7 \%$ e o segundo $12.4 \%$ da amostra, valores mais altos do que os encontrados nesse estudo. Já outro estudo dos EUA (Fernandes \& Lofgren, 2011) apresentou 28\% e 7.7\% da amostra com 1 e 2 componentes da SM alterados, resultados inferiores aos encontrados nesse estudo quando se refere a 1 e 2 componentes da SM alterados. Como já foi citado, com 3 componentes da SM alterados houve $0.81 \%$ da amostra, e não foi encontrado nenhum participante com 4 ou 5 componentes da SM alterados.

Estes resultados demonstram que os componentes da SM vêm se instalando entre as populações mais jovens da sociedade. Cada componente pode ocorrer de forma variada e ocasionar danos irreversíveis a estes jovens.

\section{Conclusão}

A prevalência da SM encontrada nessa pesquisa foi baixa, porém ao analisar os componentes individuais da SM notase uma alta prevalência do perfil lipídico alterado dos universitários (TAG e HDLc). Portanto, o planejamento para um diagnóstico precoce, como o monitoramento dos parâmetros de SM, incentivo à prática de atividade física e a adoção de alimentação saudável são importantes passos para reduzir a prevalência dos componentes da SM entre os jovens universitários.

Estudos longitudinais futuros com o objetivo de averiguar a incidência dos componentes da SM, e que envolva questões sobre o nível de sedentarismo e hábitos alimentares poderiam esclarecer melhor o surgimento de tais patologias.

\section{Referências}

Aboufotouth, M. A., Al-Alwan, I., \& Al-Rowaily, M. (2021) Prevalence of metabolic abnormalites and association with obesity among Saudi college students. Int. J. hypertents. v. 2012. https://doi.org/10.1155/2012/819726

Associação Brasileira para o Estudo da Obesidade e da Síndrome Metabólica. (2016). Diretrizes brasileiras de obesidade 2016, (4a ed.), https://abeso.org.br/wpcontent/uploads/2019/12/Diretrizes-Download-Diretrizes-Brasileiras-de-Obesidade-2016.pdf

Barbalho, S. M., Machado, F. M. F. V., Oshiiwa, M., Tomazella, P., Goulart, R. A., Meneguim, G. A. O. \& Pereira, L. L. (2009) Comparação da prevalência de fatores de risco de síndrome metabólica entre homens e mulheres acadêmicos de uma instituição pública de nível superior de Marília-SP. Revista Saúde e Pesquisa, 2 (3), 345-348. https://periodicos.unicesumar.edu.br/index.php/saudpesq/article/view/1150/894

Bicalho, J. M. F., Guimarães, E. A. A., Freitas, P. P. de, Lopes, M. S., Menezes, M. de C., Lopes, A. C. S. \& Oliveira, C. di L. (2021a). Desenvolvendo modelos para o Programa de Promoção da Alimentação Adequada e Saudável: um estudo de avaliabilidade. Research, Society and Development, 10(10), e600101019051. https://doi.org/10.33448/rsd-v10i10.19051

Bicalho, J. M. F., Sevalho, G. \& Guimarães, E. A. A. (2021b). Mudança de hábitos após diagnóstico de Diabetes Mellitus Tipo 2: Pesquisa Qualitativa. Revista Científica Multidisciplinar Núcleo do Conhecimento. 4 (5), 30-51. https://www.nucleodoconhecimento.com.br/saude/mudanca-de-habitos

Borges Neto, J. de S., Bicalho, J. M. F., Silva, T. M. da, Vieira, M. S., Bila, W. C., Freitas, P. H. B. de, Machado, R. M., \& Granjeiro, P. A. (2021). Frequência da síndrome metabólica em pacientes esquizofrênicos. Research, Society and Development, 10(11), e93101118904. https://doi.org/10.33448/rsd-v10i11.18904

de Freitas, R. W., Jr, de Araújo, M. F., Marinho, N. B., de Vasconcelos, H. C., Lima, A. C., Pereira, D. C., Almeida, P. C., Zanetti, M. L., \& Damasceno, M. M. (2013). Prevalence of the metabolic syndrome and its individual components in Brazilian college students. Journal of clinical nursing, 22(9-10), 1291-1298. https://doi.org/10.1111/jocn.12015

De Marco, M., de Simone, G., Izzo, R., Mancusi, C., Sforza, A., Giudice, R., Trimarco, B., \& De Luca, N. (2012). Classes of antihypertensive medications and blood pressure control in relation to metabolic risk factors. Journal of hypertension, 30(1), 188-193. https://doi.org/10.1097/HJH.0b013e32834e1eda

DiBello, J. R., McGarvey, S. T., Kraft, P., Goldberg, R., Campos, H., Quested, C., Laumoli, T. S., \& Baylin, A. (2009). Dietary patterns are associated with metabolic syndrome in adult Samoans. The Journal of nutrition, 139(10), 1933-1943. https://doi.org/10.3945/jn.109.107888

Eckel, R. H., Grundy, S. M., \& Zimmet, P. Z. (2005). The metabolic syndrome. The lancet, v. 365, n. 9468, p. 1415 - 1428. doi: 10.1016/S0140-6736(05)663787.

Evaristo, A. A. C. F., Medeiros, K. G., Schwingel, P. A., Lima Júnior, C., \& Lima, R. L. F. A. (2021). Risk for cardiovascular diseases in university students: Factors related to diabetes mellitus. Brazilian Journal of Development, 7(2), 18659-18673. https://www.brazilianjournals.com/index.php/ BRJD/article/view/25146/20043 
Expert Panel on Detection, Evaluation, and Treatment of High Blood Cholesterol in Adults (2001). Executive Summary of The Third Report of The National Cholesterol Education Program (NCEP) Expert Panel on Detection, Evaluation, And Treatment of High Blood Cholesterol In Adults (Adult Treatment Panel III). JAMA, 285(19), 2486-2497. https://doi.org/10.1001/jama.285.19.2486

Fernandes, J., \& Lofgren, I. E. (2011). Prevalence of metabolic syndrome and individual criteria in college students. Journal of American college health: J of ACH, 59(4), 313-321. https://doi.org/10.1080/07448481.2010.508084

Gesteiro, E., Megía, A., Guadalupe-Grau, A., Fernandez-Veledo, S., \& Vendrell, J. (2021). Early identification of metabolic syndrome risk: A review of reviews and proposal for defining pre-metabolic syndrome status. Nutrition, metabolism, and cardiovascular diseases: NMCD,31(9), $2557-2574$. https://doi.org/10.1016/j.numecd.2021.05.022

Gonçalves, R., Mendes, R. C., de Paula Símola, R. Á., Oliveira Damasceno, V., Alves Lamounier, J., \& Granjeiro, P. A. (2021). Prevalence of metabolic syndrome in Brazilian children using three different sets of international criteria. Prevalencia del síndrome metabólico en niños brasileños utilizando tres diferentes criterios internacionales. Nutricion hospitalaria, 38(2), 228-235. https://doi.org/10.20960/nh.03224

Granjeiro, P. A., da Silva, T. M., Dos Santos, D. D. R., Vieira, M. de S., Borges Neto, J. de S., \& dos Santos, M. E. S. M. (2016). Frequency of metabolic syndrome in children and adolescents from public schools of Divinópolis, Minas Gerais, Brazil, according to three international diagnostic criteria. Scientia Medica, 26(3), ID22854. https://doi.org/10.15448/1980-6108.2016.3.22854

Huang, T. T., Shimel, A., Lee, R. E., Delancey, W., \& Strother, M. L. (2007). Metabolic Risks among College Students: Prevalence and Gender Differences. Metabolic syndrome and related disorders, 5(4), 365-372. https://doi.org/10.1089/met.2007.0021

Jesus, L. F., Silva, A. O., Lima, P. F. S., Guerra, M. F., Morais, M. J., Borges Neto, J. S., Silva, O. G., Rodrigues, M. A. M., Sílio, L. F., Vilela Junior, G. B., \& Santos, D. A. T. (2020). Consumo de álcool e tabaco de estudantes de uma universidade pública. Revista Centro de Pesquisas Avançadas em Qualidade de Vida, 12 (2). https://doi.org/10.36692/cpaqv-v12n2-13

Lopes, H. F. (2007). Síndrome metabólica: uma abordagem multidisciplinar. Atheneu.

Ministério da Saúde (BR). 16,4\% dos brasileiros praticam atividade física. 2010. http://portal.saude.gov.br/portal/aplicacoes/reportagensEsp eciais/default.cfm?pg=dspDetalhes\&id_area=124\&CO_NOTICIA=10081 .

Morrell, J. S., Lofgren, I. E., Burke, J. D., \& Reilly, R. A. (2012). Metabolic syndrome, obesity, and related risk factors among college men and women. Journal of American college health: $J$ of ACH, 60(1), 82-89. https://doi.org/10.1080/07448481.2011.582208

Noel, S. E., Newby, P. K., Ordovas, J. M., \& Tucker, K. L. (2009). A traditional rice and beans pattern is associated with metabolic syndrome in Puerto Rican older adults. The Journal of nutrition, 139(7), 1360-1367. https://doi.org/10.3945/jn.109.105874

Oliveira, R. M. S., Franceschini, S. C. C., Rosado, G. P., \& Priore, S. E. (2008). Influência do Estado Nutricional Pregresso sobre o Desenvolvimento da Síndrome Metabólica em Adultos. Arquivos Brasileiros de Cardiologia, 92 (2), 107-112. https://doi.org/10.1590/S0066-782X2009000200006

Oviedo, G., de Salim, A. M., Santos, I., Sequera, S., Soufrontt, G., Suárez, P., \& Arpaia, A. (2008). Factores de riesgo de enfermedades crónicas no ransmisibles en estudiantes de la carrera de Medicina. Universidad de Carabobo, Venezuela. Año 2006 [Risk factors of nontransmissible chronic diseases in students of medicine of Carabobo University. Venezuela. Year 2006]. Nutricion hospitalaria, 23(3), 288-293.

Pereira, M. G. (2011). Artigos Científicos: como redigir, publicar e avaliar. Guanabara Koogan.

Pereira, D. C. R. (2012). Análise da circunferência do pescoço como marcador para síndrome metabólica em estudantes de uma Universidade pública de Fortaleza - CE [Dissertação de Mestrado]. Fortaleza (CE): Programa de Pós-Graduação em Enfermagem da faculdade de Farmácia, Odontologia e Enfermagem da Universidade Federal do Ceará, 113p.

Pinto, J. A. F., Granjeiro, P. A., Santos, L. L., \& Machado, R. M. (2018). Prevalence of polymorphisms in the ANKK1, DRD2, DRD3 genes and metabolic syndrome in refractory schizophrenia. Revista Latino-Americana de Enfermagem, 26. http://dx.doi.org/10.1590/1518-8345.2222.2983

Rashid, A. A., Parastouei, K., \& Shahaboddin, M. E. (2012). Metabolic syndrome among medical university students in Kashan, Iran. Scientific Research and Essays, 7 (41), 3549-3553. https://doi.org/10.5897/SRE12.204

Santos, A. K. G. V., Reis, C. C., Chaud, D. M. A., \& Marimoto, T. M. (2013). Qualidade de vida e alimentação de estudantes universitários que moram na região central de São Paulo sem a presença dos pais ou responsáveis. Revista. Simbio-Logias, 7(10). https://www1.ibb.unesp.br/Home/Departamentos/Educacao /Simbio-Logias/qualidade_de_vida_alimentacao_de_estudantes.pdf

Santos, D. A. T., Jesus, L. F., Borges Neto, J. S., Morais, M. J., Lima, P. F. S., Viana, R. B., Lira, C. A. B, Silva, W. F., \& Silva, A. O. (2019). Condutas de saúde em acadêmicos de educação física. Revista Movimenta, 12 (2),182-192. https://www.revista.ueg.br/index.php/movimenta/article/view/7425/6598

Silva, A. R. V., Sousa, L. S. N., Rocha, T. S., Cortez, R. M. A., Macêdo, L. G. N. M., \& Almeida, P. C. (2014). Prevalência de componentes metabólicos em universitários. Revista Latino-Americana de Enfermagem, 22(6). https://doi.org/10.1590/0104-1169.0129.2514

Siqueira, A. F. A., Abdalla, D. S. P., \& Ferreira, S. R. G. (2006) LDL: da síndrome metabólica à instabilização da placa aterosclerótica. Arquivos Brasileiro de Endocrinologia e Metabolismo, 50 (2): 334-43. https://doi.org/10.1590/S0004-27302006000200020

Smith, C., \& Essop, M. F. (2009). Gender differences in metabolic risk factor prevalence in a South African student population. Cardiovascular journal of Africa, 20(3), 178-182. https://www.ncbi.nlm.nih.gov/pmc/articles/PMC3721443/

Teixeira, A. C., Oliveira, C. L., Paiva, M. J. N., Santos, M. E. M. S., Rodrigues, M. A. H., Petri-Hahas, E., Granjeiro, P. A. (2014). Prevalência de síndrome metabólica em mulheres acima de 40 anos de Divinópolis-MG. Revista de Enfermagem do Centro Oeste Mineiro, 3(4):1349-1358. http://www.seer.ufsj.edu.br/index.php/recom/article/view/469

Topè, A. M., \& Rogers, P. F. (2013). Metabolic syndrome among students attending a historically black college: prevalence and gender differences. Diabetology \& metabolic syndrome, 5(1), 2. https://doi.org/10.1186/1758-5996-5-2 